\title{
Autopsie du traitement naturel du Covid-19
}

\author{
P. Goetz \\ (C) Lavoisier SAS 2020
}

Face au Covid-19 (ou SRASCov-2) qui apparaît en 2019 et qui est un virus à $A R N$, il a été rapidement important de comprendre la physiopathologie de cette atteinte virale de l'organisme humain. Quelques semaines après le démarrage de l'épidémie de ce virus, une première étude en langue chinoise [1] montrait qu'il pouvait exister des plantes de la médecine traditionnelle chinoise (MTC) freinant cette atteinte virale et mettait à l'honneur de pipérolactam, le rohitukine (alcaloïde de chromane) et l'OrgC (Leu 718, protéine codée dans le cluster de gènes T3SS). Du point de vue pharmacologique, il a été important de trouver des points d'ancrage du virus sur des tissus épithéliaux et endothéliaux de l'organisme humain pour déterminer des criblages de plantes contre ces systèmes de relation hôte-virus [2,3].

On a rapidement pu déceler que ce virus à ARN contient 13 gènes et codent 14 protéines [2] :

- protéine N : qui s'associe à l'ARN viral lors de l'assemblage du virion et permet, ce faisant, l'empaquetage du génome à l'intérieur de celui-ci ;

- protéine E : la composante principale de l'enveloppe virale ;

- protéine $\mathrm{N}$ : sa protéine de matrice est semblable à celle retrouvée dans les autres coronavirus, comportant un domaine transmembranaire à trois passages ainsi qu'une longue partie carboxy-terminale qui peut interagir avec la protéine de nucléocapside ;

- protéine S : qui comporte deux sous-unités (S1 et S2). La S1 reconnaît comme récepteur l'enzyme de conversion de l'angiotensine-2 (ACE-2) mais aussi d'autres récepteurs tels que la lectine de type C CD209L (L-SIGN) qui a un tropisme pour les ACE-2 négatifs.

Les récepteurs à $\mathrm{ACE}-2$ sont donc des cibles pour une intervention thérapeutique.

Le SRAS-CoV-2 se lie à l'enzyme ACE-2 qui est présente sur les cellules non immunes, telles que les cellules épithéliales respiratoires et intestinales, les cellules endothéliales, les cellules rénales (tubules rénaux) et les neurones céré-

\section{P. Goetz $(\square)$}

DU de phytothérapie, Paris-XIII,

F-93017 Bobigny cedex, France

e-mail : goetz.correpondance@gmail.com braux et les cellules immunitaires, comme les monocytes alvéolaires et macrophages. Il a une affinité alternative plus faible pour les autres récepteurs comme le CD209L ou la molécule d'adhésion intercellulaire spéciale non-intégrine (SIGN) du foie-ganglion lymphatique (SIGN) et la cellule dendritique (DC)-SIGN sont des récepteurs alternatifs pour le SRAS-CoV, mais avec une affinité plus faible.

On sait que l'ACE-2 protège contre l'insuffisance pulmonaire aiguë sévère et fonctionne comme un régulateur négatif du système rénine-angiotensine (SAR). Il est bien connu que l'angiotensine 2 (ANG 2) via l'activation de l'AT1R (récepteur de type 1 de l'ANG 2) favorise les effets néfastes sur l'hôte, tels que la vasoconstriction, la génération d'espèces réactives de l'oxygène, l'inflammation et le remodelage de la matrice.

Hanff et al. [4] ont discuté de l'association possible entre la mortalité cardiovasculaire associée au Covid-19 et la dérégulation du système rénine-angiotensine (SAR). D'un point de vue pharmacologique, l'inhibition du SAR conduit à une régulation positive de l'ACE-2, atténuant ainsi le syndrome respiratoire aigu et la myocardite chez les patients infectés par Covid-19. À l'inverse, l'augmentation de l'expression de l'ACE-2 peut faciliter l'accès à l'hôte de Covid-19, aggravant ainsi le tableau clinique.

En outre, la forme cristallisée de la principale protéase SARS-CoV-2 (protéase principale Mpro) est une nouvelle cible thérapeutique. Cette étude a été menée pour évaluer l'efficacité des composés bioactifs à base de plantes médicinales contre Covid-19 Mpro par une étude d'amarrage moléculaire.

Parmi les substances pouvant inhiber cet amarrage, nous trouvons des éléments susceptibles d'être plus efficaces que l'antiviral nelfinavir. Il s'agit de l'hespéridine, la rutine, la diosmine, l'apiine, la diacétylcurcumine, la 6'diméthoxyacrylophénone [5].

Parmi les inhibiteurs de la 3CL protéase (Mpro), on peut compter le pentapeptide Aster A, la ligustrazine (dans l'aliment japonais appelé nattō [fait de soja fermenté avec le Bacillus subtilis var. natto], l'acide salvianolique B (que contient Salvia miltiorrhiza), et le gingembre (Zingiberis officinalis), la racine d'Aster tartaricus L. f., racine et rhizome de Notoginseng, rhizome de livèche du Szechuan (Chuanxiong), 
racine et rhizome de Salvia miltiorrhiza Bunge, herbe de Dianthus superbus L., racine et rhizome de Rheum palmatum, herbe de cistanches. Alors que 11294 ingrédients actifs potentiels ont été obtenus par criblage d'inhibiteurs de la papain-like protease, les ingrédients actifs représentatifs comprenaient le gingékétophénol, l'alcool de ginkgol, l'acide férulique, etc., et racine de Codonopsis pilulosa, racine et rhizome de Notopterygium incisum, gingembre, graine de Ginkgo, rhizome de Ligusticum (livèche du Szechuan Chuanxiong), fruit du Trichosanthis kirilowii, racine de Paeoniae lactiflora, fruit de Psoralea corylifolia, racine de Sophora flavescens, racine et rhizome de Notoginseng, racine d'Angelica sinensis. En combinant le diagnostic et le schéma de traitement de la province du Hunan et des inhibiteurs de l'enzyme de conversion 2 de l'angiotensine (ACE-2), on obtient des herbes candidates à cette situation épidémique : fruit du Trichosanthis kirilowii, obtenu par criblage d'inhibiteurs de papain-like protease et Fritillaria verticillata obtenu par criblage d'inhibiteurs ACE-2 faisaient partie de la poudre Sang bei Zhisou et de la décoction Xiao xian xiong connus comme traitant les syndromes de toux et de dyspnée.

Parmi les plantes de la MTC qui ont activité antivirale, antibactérienne et antifongique, Joshi et al. [6] ont ciblé celles agissant sur le Mpro et l'ACE-2. Ce sont le Curcuma longa, le poivre noir (Piper nigrum) et le bétel (Piper betle Linn.) par effet du pipérolactame [7], certains flavonoïdes de Phaseolus vulgaris, le faux basilic (Ocimum gratissimum), le 3-0-caffeoylquinique du giroflier (Syzygium aromaticum), l'absinthe (Artemisia absinthium) et des polyphénols de l'aunée (Inula helenium).

Par ailleurs, nous apprenons que la physiopathologie du Covid-19 inclut une atteinte par des médiateurs comme l'interleukine IL-6. Le remède chinois Lianhua Qingwen (LHQ) a été tenté contre l'infection au Coronavirus et il a inhibé de manière significative la réplication du SRASCoV-2 dans les cellules Vero E6 et a considérablement réduit la production de cytokines pro-inflammatoires (TNF- $\alpha$, IL-6, CCL-2 / MCP-1 et CXCL-10 / IP-10) au niveau de l'ARNm [8]. De plus, le traitement à la LHQ a entraîné une morphologie anormale des particules de virion dans les cellules. Ce remède contient du fruit de Forsythia suspensa (Thunb.) Vahl $225 \mathrm{~g}$, tige d'Ephedra sinica Stapf $85 \mathrm{~g}$, bourgeon fleural de Lonicera japonica Thunb. $255 \mathrm{~g}$, racine de Isatis indigotica Fortune $255 \mathrm{~g}$, le menthol de Mentha haplocalyx Briq.7,5 g, le rhizome de Dryopteris crassirhizoma Nakai 255 g, rhizome de Rhodiola rosea L. 85 g, Gypsum fibrosum 255 g, Pogostemon cablin (Blanco) Benth. ou patchouli $85 \mathrm{~g}$, rhizome de Rheum palmatum L. $51 \mathrm{~g}$, plante entière de Houttuynia cordata Thunb. 255 g, rhizome de Glycyrrhiza uralensis Fisch. $85 \mathrm{~g}$ et graine d'Armenaria sibirica (L.) Lam. 55 g.

L'actualité et l'urgence sanitaire ont poussé les chercheurs à faire des recherches dans toutes les substances connues de la nature. Il est vrai que la MTC est riche en éléments naturels pouvant avoir un effet thérapeutique. De ce fait, les publications touchent surtout la zone extrême-orientale. Selon la légende, un médecin de renom mongol envoya ses élèves en forêt pour ramener toutes les substances n'ayant pas d'effet thérapeutique. Seul un en revint disant que toutes celles trouvées étaient utilisables dans des préparations de remèdes. Ce fut l'élu. Au XxI siècle, la médecine et la phytothérapie ne se contentent pas de légende, mais nécessitent des expérimentations incluant un contrôle clinique versus placebo. Nous sommes loin de la légende, mais l'enquête des ciblages et les croisements pourraient nous faire découvrir la perle rare qui nous manque cruellement.

\section{Références}

1. Niu M, Wang RL, Wang ZX, et al (2020) Rapid establishment of traditional Chinese medicine prevention and treatment of 2019$\mathrm{nCoV}$ based on clinical experience and molecular docking. Zhongguo Zhong Yao Za Zhi 45:1213-8

2. Ma J, Huo XQ, Chen X, et al (2020) Study on screening potential traditional Chinese medicines against 2019-nCoV based on Mpro and PLP. Zhongguo Zhong Yao Za Zhi 45:1219-24

3. Magrone T, Magrone M, Jirillo E (2020) Focus on receptors for coronaviruses with special reference to angiotensin-converting enzyme 2 as a potential drug target - A perspective. Endocr Metab Immune Disord Drug Targets 10.2174/1871530320666200427112902

4. Hanff TC, Harhay MO, Brown TS, et al (2020) Is there an association between Covid-19 mortality and the renin-angiotensin system-a call for epidemiologic investigations. Clin Infect Dis ciaa329

5. Sevki A, Volkan E, Iqra S, et al (2020) Identification of potent Covid-19 main protease (Mpro) inhibitors from natural polyphenols: an in silico strategy unveils a hope against corona. Preprints 202003033

6. Joshi T, Joshi T, Sharma P, et al (2020) In silico screening of natural compounds against Covid-19 by targeting Mpro and ACE-2 using molecular docking. Eur Rev Med Pharmacol Sci 24:452936

7. Amin SA, Bhattacharya P, Basak S, et al (2017) Pharmacoinformatics study of piperolactam a from piper betle root as new lead for non-steroidal antifertility drug development. Comput Biol Chem 67:213-24

8. Ding Y, Zeng L, Li R, et al (2017) The Chinese prescription Lianhua Qingwen capsule exerts anti-influenza activity through the inhibition of viral propagation and impacts immune function. BMC Complement Altern Med 17:130 